\title{
Ezrin/Exocyst Complex Regulates Mucin 5AC Secretion Induced by Neutrophil Elastase in Human Airway Epithelial orls
}

Qi Lia Na Lia Chun-Yi Liu ${ }^{a}$ Rui Xua Victor P. Kolosov ${ }^{b}$ Juliy M. Pe Xiang-Dong Zhou ${ }^{\text {a }}$

aDepartment of Respiratory Medicine, The Second Affiliated Hospit 'Chonga Yedical University, Chongqing, China; ${ }^{b}$ Far Eastern Scientific Center of Physiology ano Branch, Russian Academy of Medical Sciences, Blagoveschensk, Rus

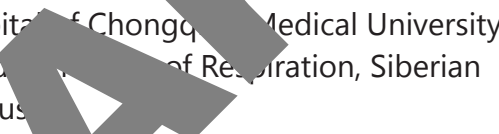

Key Words

Ezrin $\cdot \operatorname{Sec} 3 \cdot$ Mucin $5 A C \cdot$ Neu+ aphil

ase Phosphatidylinositol 4,5-bisphosphate

\section{Abstract}

Background/Aim: Increas nucin retion is a characteristic feature of many chronic airway diseases, particul auriry periods of exacerbation; however, the exact mechanism of mucin secretion reme un ar. Ezrin, which is a specific marker of apical membranes, is predominantly constin surface structures, crosslinking the actin cytoskeleton wit' an sma membrane. In the present study, we examined whether Ezrin is involved in n cir st (UUC5AC) secretion after neutrophil elastase (NE) attack, and we investigated e $r$ the exocyst complex docking protein Sec3 in this process. Methods: $\mathrm{NE}$ was $\mathrm{L}$ as a strmulator in a 16HBE140- cell culture model. The expression and location of na 3 were investigated, and the interaction between Ezrin and Sec3 in 16HBE140cel assa, ad after treatment with NE, Ezrin siRNA, Sec3 siRNA, neomycin or PIP2-Ab. Result. found that Ezrin was highly expressed in the bronchi of humans with chronic $y$ diseases. NE induced robust MUC5AC protein secretion. The Ezrin siRNA, Sec3 siRNA, d. omycin treatments led to impaired MUC5AC secretion in cells. Both Ezrin and Sec3 were in ted primarily to the cytoplasmic membrane after NE stimulation, and the neomycin and 2-Ab treatments abrogated this effect. Immunoprecipitation analysis revealed that Ezrin and Sec3 combined to form complexes; however, these complexes could not be detected in Ezrin $_{\triangle 1-333}$ mutant-transfected cells, even when PIP2 was added. Conclusions: These results demonstrate that Ezrin/Sec3 complexes are essential for MUC5AC secretion in NE-stimulated airway epithelial cells and that PIP2 is of critical importance in the formation of these complexes. 


\section{Introduction}

Physiologically secreted mucus is an important defense barrier in the respiratory system. Mucus can remove foreign substances and maintain the airway microenvironment $[1,2]$. However, excess mucus is recruited to the airway during acute exacerbations of chronic obstructive pulmonary disease and severe asthma, particularly during fatal asthm attacks [3-5]; typically, a "burst" of mucus production occurs, forming a mucus plug tha can block the airway such that the patient can die of asphyxiation. Studies have found that many factors, such as bacteria, viruses, inflammatory factors and proteases, can in mucin synthesis and promote exocytosis. These factors simultaneously activate the sig cascade involved in exocytosis [6-8]. Therefore, we hypothesized that the under pathological conditions may strongly correlate with mucus exocytos understanding the signaling mechanisms involved in exocytosis may lead for sputum retention, thus preventing airway obstruction.

Ezrin is a specific marker of apical membranes. This protein li actin of the cytoskeleton to the apical membrane of cells. Ezrin is p arily aruibuted in organs that have secretory functions, such as lungs, small intestine, s ch, pancreas, and kidneys, and it is highly expressed in the epidermis and This protein is predominantly concentrated in actin-rich cell surface struct sa. yolved in microvilli formation, cell adhesion, cell motility and secretion [10].

The exocyst is an octameric protein complex involved in ering post-Golgi secretory vesicles to the plasma membrane. Sec3 is a do protein o ne exocyst complex that is required for secretion. The N-terminus of the exod omponent Sec3 directly interacts with phosphatidylinositol 4,5-bisphosphate (PIP2), on nd PIP combine to play important roles in polarized cell growth and exocytosis. evic study also found that ERM (Ezrin/ Radixin/Moesin) proteins target sites in nembrane that are enriched in lipid rafts and exocyst complexes [11]. Ho ve whether Ezrin and Sec3 interact to regulate mucin secretion in airway epithelial ce $\mathrm{n}$ s unclear.

Chronic respiratory disea con hypersecretion are usually accompanied by marked neutrophil infiltrati ticularly in cases of fatal asthma. This situation differs from that of conventional asthma, is characterized by eosinophil infiltration $[12,13]$. Neutrophil elastase (NE) Crated by neutrophils has been shown to be a potent mucin secretagogue [14]. In t pres study, we report that NE exposure increases MUC5AC secretion and that Ezrin exocyst complex subunit Sec3 are involved in the secretion process. The dat proviue the first evidence that Ezrin and Sec3 function together and play impor ti in mucus secretion in airways. Our study reveals the molecular mechanism f ex vs complex formation and improves our understanding of mucin secretion fflan ory airway diseases.
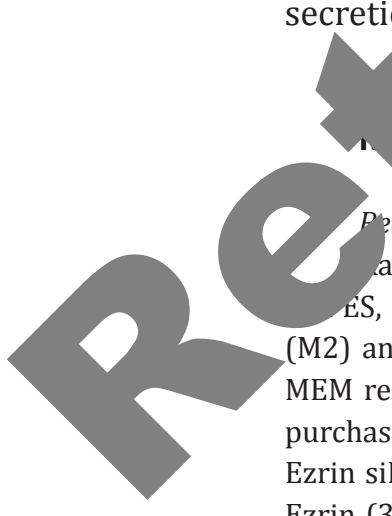
(M2) antibody were purchased from Sigma-Aldrich (St. Louis, MO, USA). Lipofectamine 2000 and OPTIMEM reduced-serum medium were purchased from Invitrogen (San Diego, CA, USA). PIP2 antibody was purchased from Abcam (Cambridge, MA, USA). Protein A/G PLUS-Agarose immunoprecipitation reagent, Ezrin siRNA, Sec3 siRNA (h2), control siRNA, rabbit anti-Ezrin (Thr567) polyclonal antibody, mouse antiEzrin (3C12) monoclonal antibody and FITC-conjugated fluorescent secondary antibodies were obtained from Santa Cruz Biotechnology (Santa Cruz, CA, USA). X-tremeGENE9 DNA transfection reagent was obtained from Roche (Roche, Basel, Switzerland). 


\section{Tissues}

Normal human lung tissues were obtained from surgical specimens resected at the Department of Thoracic Surgery of the Second Affiliated Hospital (Chongqing Medical University) between 2011 and 2012. The experiments were approved by the institutional ethics committee, and informed consent was obtained before sampling. The samples were collected from 10 patients ( 8 men and 2 women) with a mean age of 56 years (age range: 45-69 years). At the time of surgery, 6 patients were suffering from mild to moderat COPD or asthma, and 4 patients had no underlying chronic inflammatory diseases of the airway. None the patients was being chronically treated with theophylline, $\beta$-adrenoceptor agonists, corticosteroids, or anticholinergic drugs. Hematoxylin and eosin (HE) staining was used to confirm whether the tissues non-cancerous.

\section{Immunohistochemistry}

Tissue slides were incubated with a mouse anti-Ezrin monoclonal antibody (1: temperature for $1 \mathrm{~h}$ and then incubated with a rabbit anti-mouse antibody 1:200 Then, Then, samples were exposed to a streptavidin-peroxidase complex (1:100 diluti, ror, 45 min. 3,3'-Diaminobenzidine (DAB) was used as a chromogen. Epithelial cells that $s$ positmuly for Ezrin were counted; the results are expressed as a percentage of the total number of ep cells.

Construction of recombinant Flag-Ezrin and a Flag-Ezrin 1-333 ca
Total RNA was extracted from 16HBE14o- cells. The following pr sequence by PCR: forward primer, 5'-GCG AAG CTT GAT TAC AAG GAC G AT GAC AAG ACC GAA ACC AAT CAA TGT C-3' (the BamH I restriction site is underline $\mathrm{d}$ the Flag st ence is in italics), and reverse primer, 5'-GCG GGA TCC TTA CAG GGC CTC GAA CTC-3 HindIII restriction site is underlined). PCR was performed using Platinum Taq DNA Polymerase cDNA. The pcDNA3.1 vector was cut at unique BamH $\mathrm{Hir}$ restriction sites, and the purified Ezrin cDNA was subcloned into the linear pcDNA3.1 DNA ligase to obtain pcDNA.3-Flag-Ezrin. The region in Ezrin that is responsible for $\mathrm{P}-\mathrm{b}$ is in the 1-333 NH2-terminal domain; thus, we constructed an Ezrin 1-333 deletion mutar ar r study. The pGEX-2T-Ezrin ${ }_{\Delta 1-333}$ plasmid used to express the Ezrin 1-333 truncated r cted as described previously [15-17]. The Ezrin ${ }_{\Delta 1-333}$ fragment was purified and subclone the pcDNA3.1-Flag vector to obtain pcDNA.3-Flag-Ezrin ${ }_{\Delta 1-333^{*}}$.

\section{Cell culture}

16HBE140- cells were ured PMI-1640 supplemented with $10 \%(\mathrm{v} / \mathrm{v}$ ) fetal bovine serum (FBS). Then, the cells were plated a of $1 \times 10^{6}-2 \times 10^{6}$ cells $/ \mathrm{ml}$ and incubated at $37^{\circ} \mathrm{C}$ until the cells were $60 \%-80 \%$ confluen were pre-treated 1

$16 \mathrm{HB}$ airway epithelial cells were plated at a density of $1 \times 10^{6}-2 \times 10^{6}$ cells $/ \mathrm{ml}$ and incubated at 37 the were $60 \%-80 \%$ confluent. Then, $4 \mu$ l of X-tremeGENE9 DNA transfection reagent was dilun was aed to the reagent. The mixture was incubated at room temperature for $20 \mathrm{~min}$. Then, the n cus was added dropwise to cells and incubated for $18 \mathrm{~h}$. Next, the cells were washed 3 times with PBS nd ubated in DMEM/Ham's F12 medium containing 10\% FBS for the following assays.

Real-time PCR and reverse transcription PCR

Total RNA was extracted from each group of 16HBE14o- cells using a Bioteke high-purity total RNA extraction kit and verified by $1.5 \%$ agarose gel electrophoresis. RNA was primed with oligo(dT) and reverse transcribed using a PrimeScript RT reagent kit. The conditions for the reverse transcription reaction were as follows: denaturing at $94^{\circ} \mathrm{C}$ for $10 \mathrm{~min}$; followed by 30 cycles of $94^{\circ} \mathrm{C}$ for $30 \mathrm{~s}, 60^{\circ} \mathrm{C}$ for $45 \mathrm{~s}$, and $70^{\circ} \mathrm{C}$ for 45 $\mathrm{s}$; and a final extension at $72^{\circ} \mathrm{C}$ for $5 \mathrm{~min}$. The PCR products were resolved by $2 \%$ agarose gel electrophoresis and subsequently visualized by ethidium bromide staining. Real-time PCR was performed using a SYBRR Premix EX Taq ${ }^{\mathrm{TM}}$ II real time PCR kit. The human Ezrin primer sequences for real-time PCR are listed in Table 1. Relative mRNA expression was determined using a standard curve.

\section{KARGER}


Table 1. Primers for PCR

\begin{tabular}{|c|c|}
\hline Gene & Primers \\
\hline Ezrin & $\begin{array}{l}\text { Forward: 5'-AAGGGTTCTGCTCTGACTCCA-3 } \\
\text { Reverse: 5'-CCGGAACTTGAACTGGAGGG-3' }\end{array}$ \\
\hline Ezrin (real-time) & $\begin{array}{l}\text { Forward: 5'-CGCTCTAAGGGTTCTGCTCT-3' } \\
\text { Reverse: 5'-AACTTGGCCCGGAACTTGAA-3' }\end{array}$ \\
\hline GAPDH & $\begin{array}{l}\text { Forward: 5'-GAAGGTGAAGGTCGGAGT-3' } \\
\text { Reverse: 5'-GAAGATGGTGATGGGATTT }\end{array}$ \\
\hline
\end{tabular}

ELISA

ELISA for the MUC5AC protein was performed as described previously [18].

Immunoprecipitation

Protein samples were lysed using RIPA buffer and then collected, and IgC specific binding. The anti-Ezrin antibody $(1 \mu \mathrm{g})$ was added to each sample and the Then, $20 \mu$ l of protein $A+G$ agarose was added, and the samples samples were centrifuged at $2500 \mathrm{pm}$ for $5 \mathrm{~min}$ at $4^{\circ} \mathrm{C}$. The pellet centrifuged, resuspended in $20 \mu \mathrm{l}$ of buffer solution, and heated at 100 were fractionated by SDS-PAGE and then transferred onto polyvinyliden immunoblotting for Flag-Ezrin and Sec3 proteins.

\section{Immunoblotting}

The proteins in cell lysates were extracted and in transfer buffer containing $25 \mathrm{mM}$ Tris-HCl, $100 \mathrm{~m}$ proteins were transferred to PVDF membre proteins were transferred to PVDF membra on - rectrophoresis. The membranes were incubated in 5\% milk dissolved in PBS and 0.05\% Tweer 1 at room temperature and subsequently incubated with pEzrin, Ezrin, and Sec3 antibo C. After incubation with secondary IgG antibody, the immunoreactivity was visualized us enhanced chemiluminescence detection reagent.

Immunofluorescence

Cells were fixed with for $30 \mathrm{~min}$. The cells were 1 with a mouse anti-F Triton X-100/PBS ef friton X-100/PBS $2 \mathrm{~h}$ at roo temp aty e, followed by staining of the cell nuclei with diamidino-phenyl-indole (DAPI). for $2 \mathrm{~h}$ at roo temp aty e, followed by staining of the cell nuclei with diamidino-phenyl-indole (DAPI). The sampl re ex d using a Leica TCS-SP2 inverted confocal microscope fitted with the appropriate fluo sona rs.

value defined as $P<0.05$.
valing paraf haldehyde and then permeabilized with $0.1 \%$ Triton X-100 in PBS ked with $1 \%$ BSA plus $1 \%$ normal goat serum and then incubated ocrunal antibody $(1: 200)$ overnight at $4^{\circ} \mathrm{C}$. After three 10 -min washes in $0.1 \%$

\section{Results}

Ezrin expression in human bronchial epithelial cells

Ezrin was highly expressed in the mucosal epithelium of bronchi affected by chronic inflammation. This protein was primarily at the apical membrane near the cavosurface (Fig. 1A-D). The brown areas (positive areas stained by Ezrin antibody) were selected as areas 
Fig. 1. Ezrin expression in the bronchial epithelia of subjects with COPD and of control subjects. Tissues were immunostained with a mouse anti-Ezrin monoclonal antibody (1:500 dilution) at room temperature for 1 $\mathrm{h}$ and then incubated with a rabbit anti-mouse antibody (1:200 dilution) for $30 \mathrm{~min}$. Then, samples were exposed to a streptavidin-peroxidase complex (1:100 dilution) for another 45 min. 3,3'-Diaminobenzidine (DAB) was used as a chromogen. Epithelial cells that stained positively for Ezrin were counted. The data were analyzed using one-way analysis of variance (ANOVA), followed by Bonferroni analysis. The results are expressed as a percentage of the total number of epithelial cells. Significance was set at $P<0.05$ (A) Normal controls, (B) COPD group, (C) negative control group (rabbit IgG), (D) blocking peptide group (rabbit Ezrin antibody-specific peptide to block the first antibody), and (E) Ezrin protein expression in the airway epithelium (IOD/ area). *: Compared with normal control group, $P<0.01$.
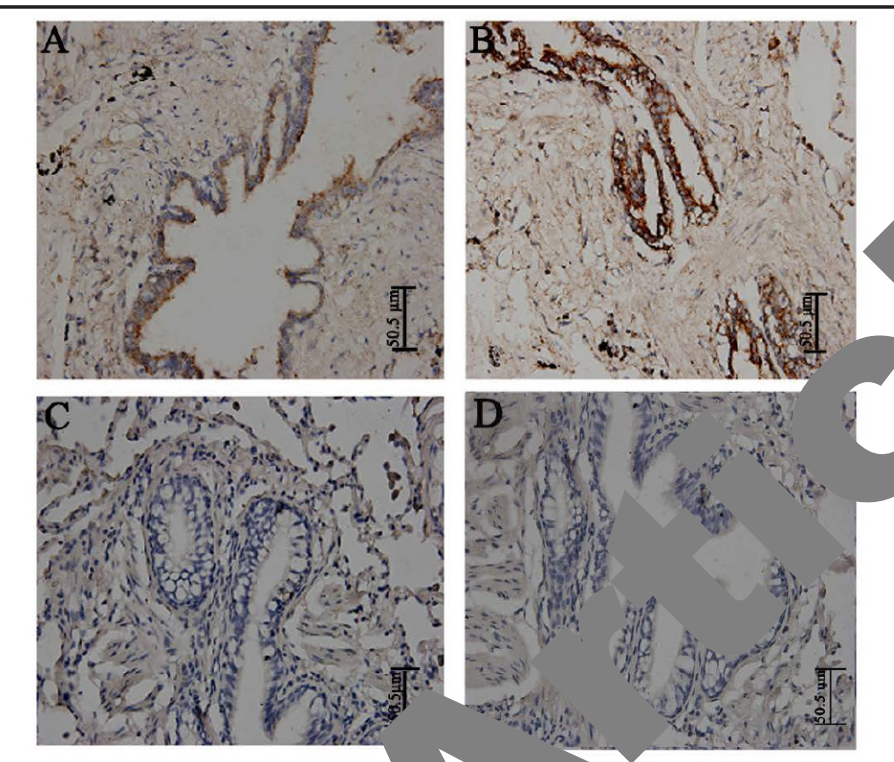

of interest (AOIs), and the integra

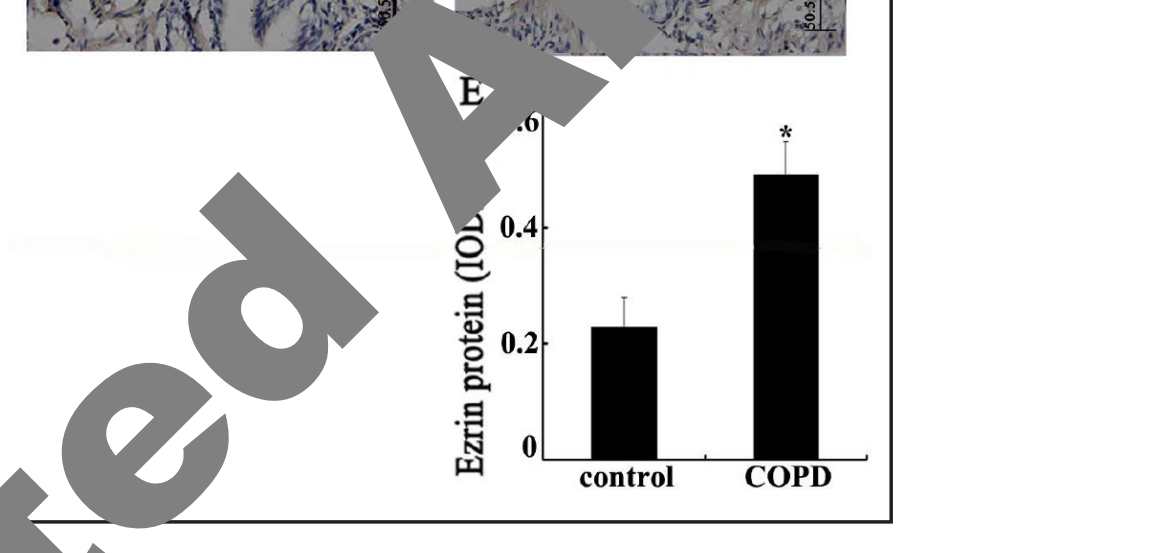

Image-Pro Plus 6.0 softy The dàt were analyzed using one-way analysis of variance (ANOVA), followed by B erro nalysis. The results revealed that the intensity (IOD/area) of the positive area for th higher than that $f$ nar prthelium samples (Fig. 1E). Thus, airway epithelial cells under chronic inflamr ic essed more Ezrin than normal epithelial cells.

NE ir ed E expression and phosphorylation in 16HBE14o-cells

Cells treated with $0.5 \mu \mathrm{M}$ NE. Ezrin mRNA expression was upregulated by NE in a tir pena manner, with the level of Ezrin mRNA expression increasing at $20 \mathrm{~min}$ and 30.4 and B). We also assayed total Ezrin and phosphorylated Ezrin (pEzrin) protein 's ù owestern blot. $\beta$-Actin was used as the loading control. The results showed that (1) Induced Ezrin phosphorylation in a time-dependent manner; the pEzrin protein level seg to increase at $5 \mathrm{~min}$ after NE incubation, and the pEzrin protein was greatly increased 0 min compared with that of the cells at time 0. Additionally, the total Ezrin protein level increased at the 30 min period (Fig. 2C and D). These results revealed that NE, which is a potent agent that leads to mucin secretion, also has the ability to increase the mRNA and protein expression levels of Ezrin and to increase the activated state of the Ezrin protein.

NE stimulated both Ezrin and Sec3 to localize to the plasma membrane

Immunofluorescence analysis showed that Ezrin was predominantly diffuse throughout the cytoplasm in normal cells without any treatment (Fig. 3A). NE stimulation led to the accumulation of Ezrin at the apical membrane (Fig. 3B). Then, we pretreated cells with $10 \mathrm{mM}$ neomycin for $30 \mathrm{~min}$ before NE stimulation. Neomycin is a positively charged aminoglycoside 

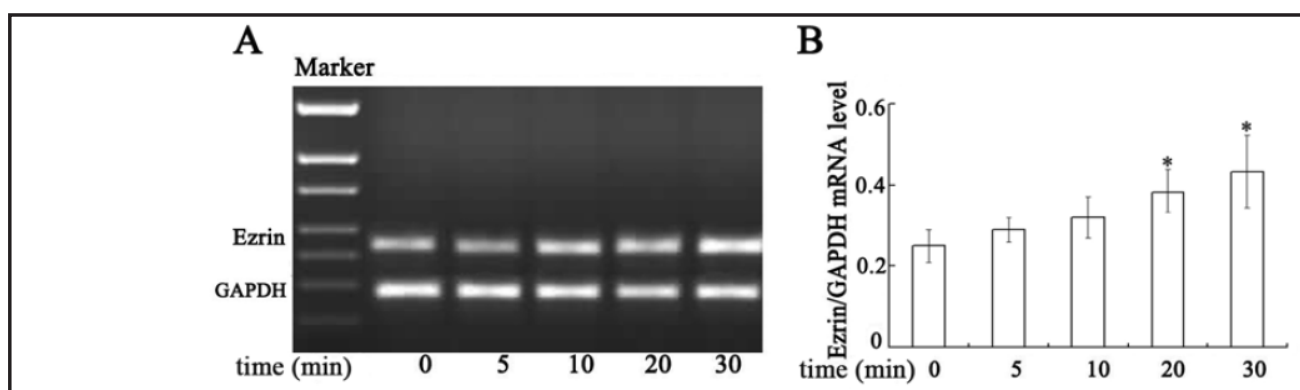

C

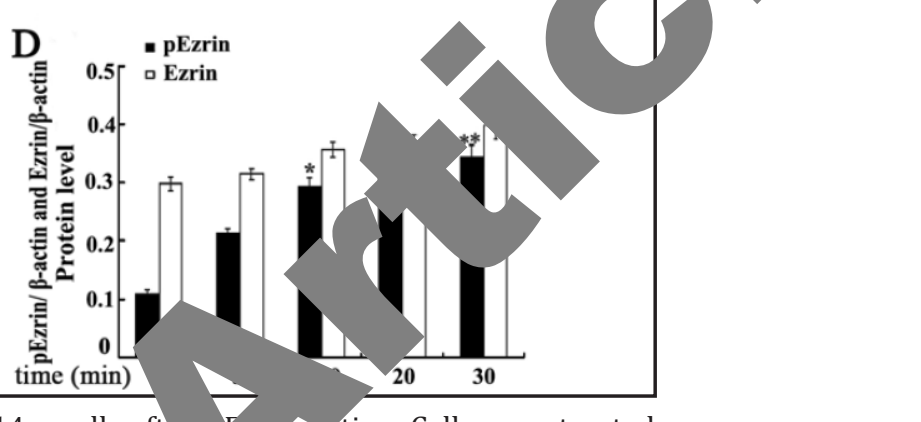

Fig. 2. Ezrin expression and phosphorylation in 16HBE14o- cells aft with $0.5 \mu \mathrm{M}$ NE. The expression of Ezrin mRNA was assayed at differen tion-PCR and real-time PCR, and the expression of Ez rotein was as ded at different time points by western blot. The data are expressed as the means \pm stanc $\quad$ ror (SE), $n=6$. (A) Ezrin mRNA and GAPDH mRNA expression were detected by reverse transcript sion were detected by real-time PCR. The results are ress s the ratio of Ezrin/GAPDH. *Compared with cells at time 0, $P<0.05$. (C) Total Ezrin protand orylated Ezrin (pEzrin) protein levels were assayed by western blot. (D) The ratios of $p^{\prime}, \mathrm{n}$ stein to $\beta$-actin protein and of total Ezrin protein to $\beta$-actin protein. *: Compared with 0 time po ${ }_{1} \mathrm{~S}, \quad 0.05 ;{ }^{* *}$ : compared with cells at time $0, P<0.01 ; \Delta$ : compared with cells at time $0, P<0$.

antibiotic known to sequester Plh high affinity. This dose of neomycin was chosen based on a previous stud formeurby Missiaen et al. [19]. In neomycin-incubated cells, Ezrin was predominant disp ed in the cytoplasm, which was a significantly different pattern compared with t NE-treated cells not exposed to neomycin (Fig. 3C and D).

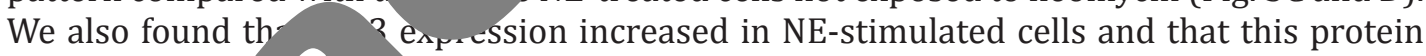
showed the san to localize to the cell surface. Neomycin treatment decreased the accumulatio or 3 . the cell surface, which is a significantly different result compared with that NE-t. d cells $(P<0.05)$ (Fig. $3 \mathrm{C}$ and D). These results demonstrated that NE co/'d stim. both Ezrin and Sec3 to localize to the cell surface.

and Sec3 levels in the plasma membrane were reduced by neomycin and by PIP2-Ab Pro S analyses of various membrane markers have shown that exocyst components a primarily found in the plasma membrane 2 (P2) fraction [20]. Therefore, the association it exocyst with the plasma membrane can be assessed by determining the level of this plex in the P2 fraction. The levels of Sec3 and Ezrin in the P2 fraction were determined by immunoblotting. Cell lysates were centrifuged successively at $500 \mathrm{~g}$ and $13000 \mathrm{~g}$ for $10 \mathrm{~min}$ to generate the S1 (cytosol fraction 1), P1 (plasma membrane fraction 1), S2 (cytosol fraction 2 ) and P2 fractions as previously described [20]. The results revealed that the levels of both Ezrin and Sec3 in the P2 fraction of NE-treated cells were higher compared with those in the P2 fraction of negative control cells $(P<0.01)$. When the PIP2-masking reagent neomycin was added, the levels of both Ezrin and Sec3 in the P2 fraction decreased compared with those of the NE-treated group; however, Ezrin and Sec3 protein levels did not show significant changes in the S1 and S2 fractions when the PIP2-masking reagent neomycin was added. Then, we treated cells with $20 \mu \mathrm{g} / \mathrm{ml}$ of PIP2 antibody (PIP2-Ab), which could reduce PIP2 
Fig. 3. Ezrin and $\mathrm{Sec} 3$ localization at the membrane was reduced by neomycin treatment. The fluorescence intensities were expressed as IOD/area. IOD/area values were calculated using Image pro-plus-6.0 software. (A) Ezrin and Sec3 expression in normal 16HBE14o- airway epithelial cells. (B) Ezrin and Sec3 expression in NE-treated cells. (C) Ezrin and Sec3 expression in cells preincubated with neomycin and then treated with NE. (D) The fluorescence intensities of Ezrin and Sec3 at the plasma membrane. $\Delta$ : Compared with control cells, $P<0.05$; *: compared with NE-treated cells, $P<0.05$.

activity in cells [21l anc



alt indicated that PIP2-Ab treatment also decreased both Ezrin and Sec3 pr vers in the P2 fraction, resulting in significant differences compared with those of $\mathrm{N}$ - ed cells $(P<0.01)$ (Fig. 4A-D). These results revealed that PIP2 might parti ate ${ }^{\text {th }}$ translocation of Ezrin and Sec3 to the plasma membrane.

MC. vrotein secretion inhibited by Ezrin siRNA, Sec3 siRNA, neomycin and PIP2-Ab Is we cransfected with Ezrin siRNA and then stimulated with NE for $30 \mathrm{~min}$. MU tein secretion into the supernatant of the cultured cells was decreased compared tha non-transfected cells; however, no obvious change in the level of MUC5AC in the C or"asm was observed (Fig. 5A). The MUC5AC protein level in the supernatant was also ed ed in Sec3 siRNA-transfected cells compared with that in non-transfected cells (Fig. s. To investigate the role of PIP2 in this process, Ezrin siRNA- or Sec3 siRNA-transfected cells were preincubated with $20 \mu \mathrm{M}$ PIP2 before NE incubation. The PIP2 dose was chosen according to the study of Kim et al. [21]. The results showed that PIP2 could increase the MUC5AC protein level in the cytoplasm but not in the supernatant of Ezrin siRNA-transfected cells after NE stimulation. An identical effect was detected in Sec3 siRNA-transfected cells preincubated with PIP2 before NE stimulation (Fig. 5A). Thus, the effect of NE on MUC5AC secretion into the supernatant was attenuated in Ezrin- and Sec3-inhibited cells, even when exogenous PIP2 was added. These results indicated that Ezrin and Sec3 were involved in NE-induced MUC5AC protein secretion into the supernatant but not in MUC5AC protein production in the cytoplasm. 
Fig. 4. The Ezrin and Sec3 levels at the plasma membrane were reduced by neomycin and PIP2Ab treatments. The data are expressed as the means \pm standard error (SE), $n=6$. (A) Ezrin protein expression in each fraction. (B) Sec3 protein expression in each fraction. S1: cytosol fraction 1; S2: cytosol fraction 2; P2: plasma membrane fraction 2. (C) The relative ratio of Ezrin protein in $\mathrm{P} 2$ and S2 fractions. *: Compared with control cells, $P<0.01$; **: compared with NE-treated cells, $P<0.01$. (D) The relative ratio of Sec3 protein in P2 and S2 fractions. *: Compared with control cells, $P<0.01$; ${ }^{* *}$ : compared with NE-treated cells, $P<0.01$.

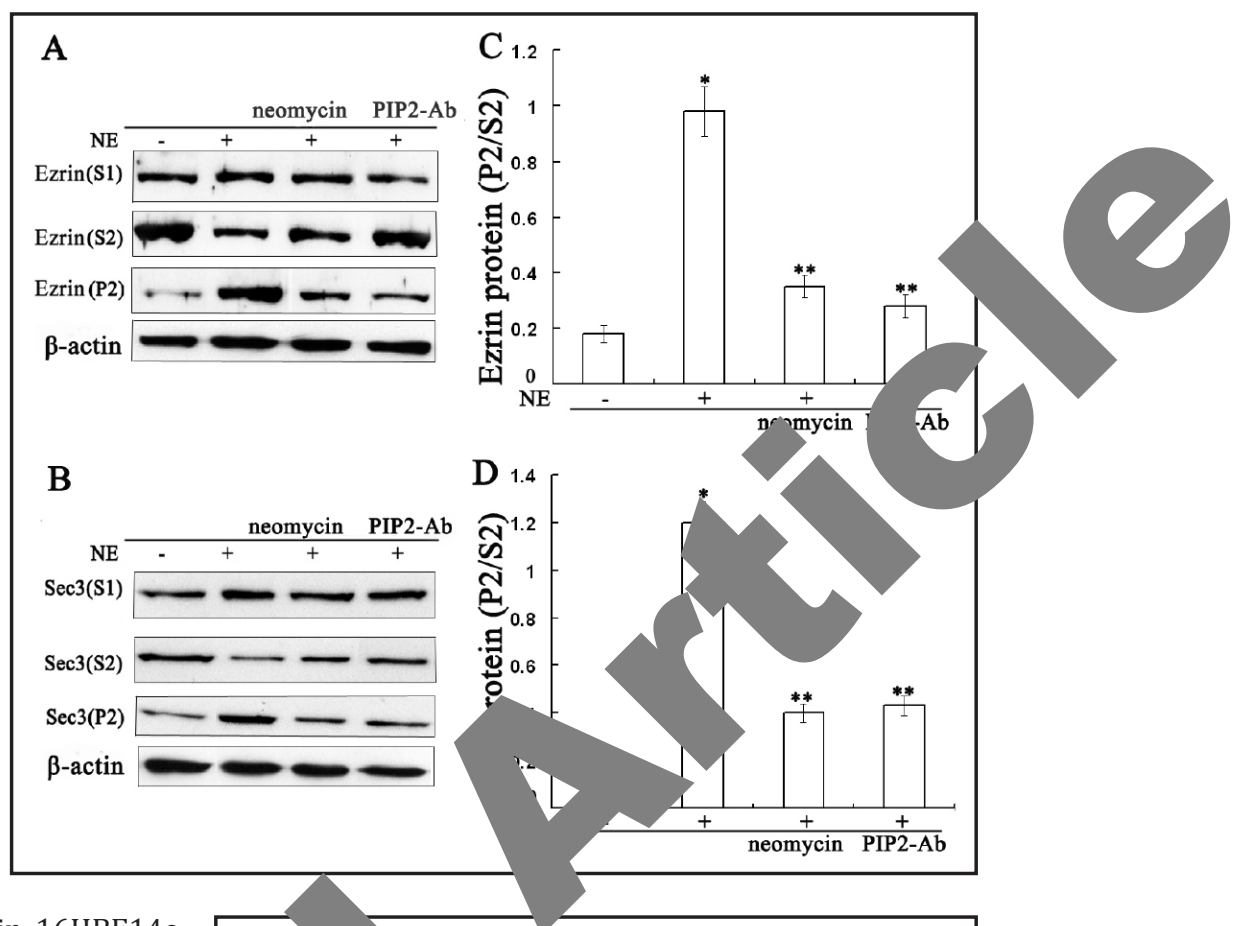

Fig. 5. MUC5AC protein levels in 16HBE14oepithelial cells and in culture supernatant. The data are expressed as the means \pm standard error (SE), $n=6$. (A) The MUC5AC protein level in the culture supernatant was decreased by transfer tion with Ezrin and Sec3 siRNAs. *: Compa with NE-treated cells, $P<0.05$. When $O$ no PIP2 was added to Ezrin siRNA- o NA-transfected cells, the MUC5AC prua in the supernatant did not incr signific...y but remained at a lower leve Com ed with NE-treated cells, $P<0.05$. Ho r, th AUC5AC protein level in the las. Compared with Ez or iRNA-transfected cells without PIPZ $<$ 05. MUC5AC protein level in the resu ant decreased when treated wic. mycin and PIP2-Ab. $\Delta$ : Comparec NE- $\mathrm{d}$ cells, $P<0.01$; *: compared wit eated vells, $P<0.05$.

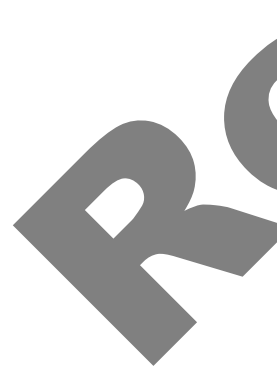
12 ह
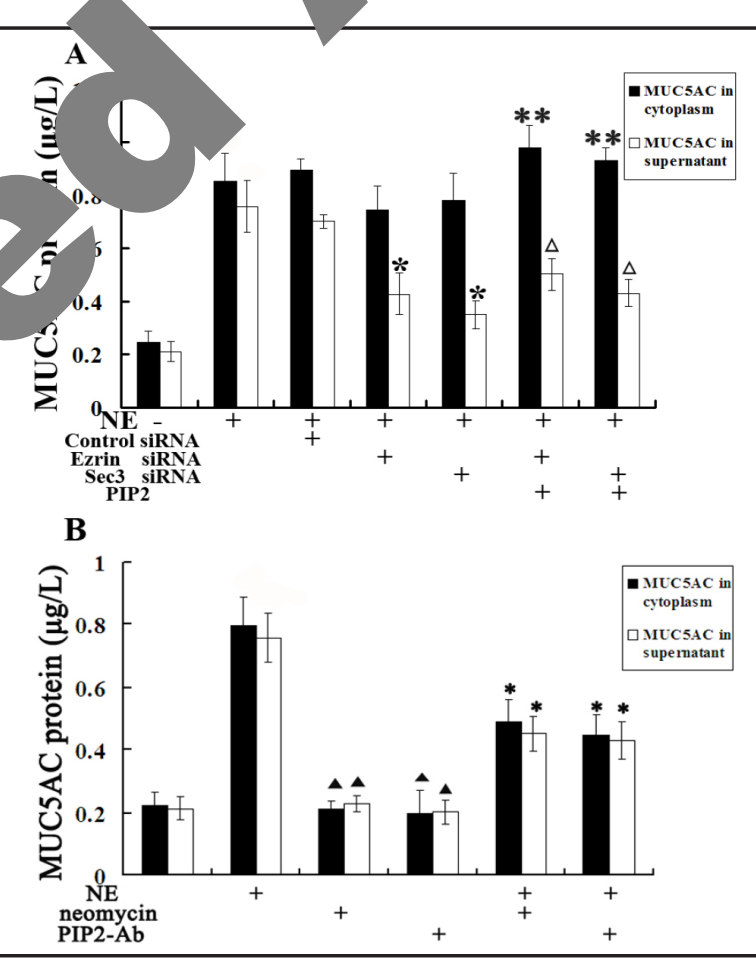

In the present study, we found that PIP2 could increase MUC5AC protein levels in the cytoplasm under NE stimulation in the Ezrin siRNA- and Sec3 siRNA-transfected cells. To further study the role of PIP2, these cells were preincubated with $10 \mathrm{mM}$ neomycin or 20 $\mu \mathrm{g} / \mathrm{ml}$ of PIP2-Ab for 30 min before NE stimulation, and the MUC5AC protein levels in the supernatant and cytoplasm were assayed. The results showed that both neomycin and PIP2$\mathrm{Ab}$ decreased the MUC5AC protein level in both the cell culture supernatant and cytoplasm (Fig. 5B).

The above results revealed that exogenous PIP2 could increase the MUC5AC protein level in the cytoplasm but not in the supernatant of Ezrin siRNA- or Sec3 siRNA-transfected 


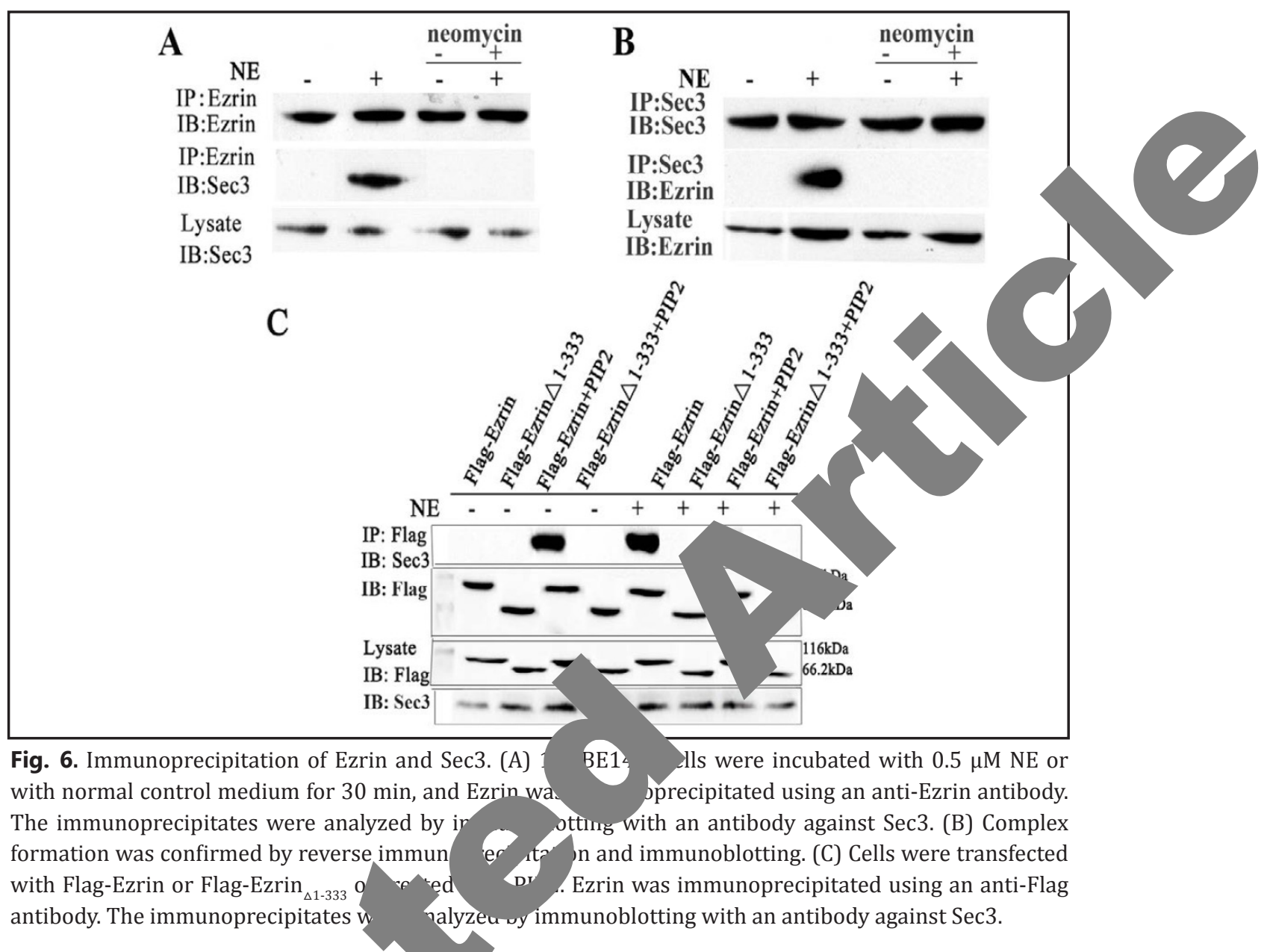

cells under NE stimula The innibition of PIP2 by neomycin and PIP2-Ab caused decreasing the MUC5AC oteif vels in both the culture supernatant and cytoplasm. The effects of neomycin and $\mathrm{on}$ the MUC5AC protein level in the cytoplasm differed from those of Ezrin si Sec siRNA. This difference may be because PIP2 is an important signal mediator a d mucin hypersecretion events initiated by many stimuli, such as proteases [? an al [23]. PIP2 may be involved in both MUC5AC protein production and secretion hese pesses.

stimu es Ezrin binding to Sec3, and PIP2 is required for Ezrin-Sec3 binding

$\mathrm{d}$ that NE stimulated both Ezrin and Sec3. We used immunoprecipitation assays ydy eir interaction further. Confluent 16HBE14o- cells were starved and stimulated (1) $5 \mu \mathrm{M}$ NE for $30 \mathrm{~min}$. Ezrin was immunoprecipitated with an anti-Ezrin antibody. The $\mathrm{m}$ moprecipitates were analyzed by immunoblotting with antibodies to Ezrin and Sec3. immunoprecipitation experiments showed that Ezrin bound to Sec3 (Fig. 6A and B). Then, we transfected cells with Flag-Ezrin or Flag-Ezrin ${ }_{\triangle 1-333}$; some cells were also treated with $20 \mu \mathrm{M}$ PIP2 for further evaluation. These cells were immunoprecipitated using a Flag antibody and then immunoblotted with a Sec3 antibody. We detected Sec3 in the samples immunoprecipitated by Flag, indicating that Ezrin and Sec3 interact under these conditions. For cells transfected with Flag-Ezrin and then stimulated with NE, the immunoprecipitation experiments showed that both Flag and Sec3 were expressed but no Sec3 protein was detected in the Flag-Ezrin ${ }_{\triangle 1-333}$-transfected cells, even when PIP2 was added (Fig. 6C). These results indicated that NE stimulates Ezrin binding to Sec3, that PIP2 is required for EzrinSec3 binding, and that the PIP2 binding site 1-333 in Ezrin is indispensable for PIP2 function. 


\section{Discussion}

Our results indicated that Ezrin was highly expressed in the bronchi of humans with chronic airway diseases. NE induced robust MUC5AC protein secretion into the supernatant and cell lysates in a 16HBE14o- cell culture model. MUC5AC secretion was impaired in culture supernatants when cells were transfected with Ezrin siRNA, and this effect wa detected in Sec3 siRNA-transfected cells and in neomycin- or PIP2-Ab-treated cells. Bot Ezrin and Sec3 were involved in MUC5AC secretion; this process primarily recruited Ezrin and Sec3 to the cytoplasmic membrane after NE stimulation, where these proteins comb: to form complexes. PIP2 is of critical importance in the formation of these complexes MUC5AC secretion.

To our knowledge, this study is the first report of Ezrin activation in muc in airway epithelial cells. Excessive mucin secretion is a prominent featur diseases and can lead to airway obstruction and bacteria colonizatio exocytosis is a complicated process that involves numerous factors, not been fully elucidated. We do not know precisely how the polarity ne mu granules develops or how the cell interacts with exocytosic-related eins. In addition, mucin stored in the cytoplasm cannot increase suddenly hav own that more mucin is stored in the epithelium per unit surface area o, Sa. in the bronchi of smokers with airflow obstruction, indicative of the dysregu. of mucin secretion [26]. Mucin tends to exhibit a burst secretion pattern under pat gical conditions or upon exposure to certain stimuli; this pattern differ m the bas attern of secretion under physiological states.

Ezrin is primarily distributed in secretory o and mesothelium. In the present study, we de bronchi from patients with chronic inflam tio. is is highly expressed in the epidermis nstr that Ezrin is highly expressed in 1, we used NE, which is a well-known potent inducer of mucin secretion, to $g$ er the hypersecretion state. NE induced robust MUC5AC protein secretion into the st at and cell lysates, which is consistent with the results of previous studie 2 MRNA expression was upregulated by NE stimulation; western blot assay ved that Ezrin activation also increased significantly and that Ezrin was translocated from th plasm to the cell membrane after NE stimulation.

The ERM family belo $\omega$ the talm and band 4.1R (erythrocyte membrane-cytoskeleton linker protein band 4.1 or $\mathrm{F}$ 41) superfamily. Ezrin is an important member of the ERM family. This protei. cally binds to actin and serves as a crosslinker between the plasma mem' na crie cytoskeleton $[28,29]$. The Ezrin (NM-003379) gene is on chromosome 6 5. and encodes 587 amino acids [30]. Amino acid residues 1-296 in the N-ter inus or a 4.1 FERM (Ezrin/Radixin/Moesin) domain, and residues 1-333 contain osph ylinositol 4,5-bisphosphate (PIP2) membrane binding site, which lea $\rightarrow$ c mational changes that stabilize the activation state of the C-terminus and wh esult he localization of Ezrin at the plasma membrane [31, 32]. Residues 29746 consecutive a-helix domain, which is called the central domain. Residues 460 are cring of proline residues, which could form a hinge region and thus could affect t $C^{\prime}$ nformational changes of Ezrin. The C-terminal ERM association domain (C-ERMAD) if $\mathrm{F}$ in is formed by amino acid residues 478-586 and is also called the autoinhibitory rminal tail, which has the ability to bind to the plasma membrane. Amino acid residues 551-586 form the F-actin binding domain [33, 34].

PIP2 can bind to Ezrin primarily through residues 253, 254, 262 and 263 in the NH2terminus [35]. PIP2 binds to Ezrin, and the phosphorylation of Thr567 is essential for Ezrin to translocate to the apical membrane $[36,37]$. We transfected cells with Ezrin siRNA to determine whether Ezrin and PIP2 are involved in NE-induced MUC5AC secretion. Ezrin siRNA transfection reduced the MUC5AC protein level in the culture supernatant but not the intracellular protein level, indicating that Ezrin was involved in NE-induced MUC5AC 
protein secretion but not in MUC5AC protein production in the cytoplasm. Then, when exogenous PIP2 was added to Ezrin siRNA-transfected cells before NE stimulation, we found that the MUC5AC protein level in the supernatant remained at a relatively lower level but that the MUC5AC protein level in the cytoplasm increased. Thus, PIP2 participated in Ezrinmediated MUC5AC protein secretion into the supernatant and might be involved in MUC5AC production in the cytoplasm, which was independent of Ezrin. This finding may be becaus PIP2 is an important signal mediator in a series of mucin hypersecretion events initiatec by many stimuli, such as proteases [22] and cold [23]. In addition, we found that neomycin, which is a PIP2-masking reagent, and PIP2-Ab led to impaired MUC5AC secretion in These results indicate that Ezrin is involved in MUC5AC secretion in 16HBE14o- ce that PIP2 binding could be necessary for this process.

The exocyst complex is essential for many exocystic events because this com vesicles to the plasma membrane for fusion [38], as we described for mu exocyst is a conserved multi-subunit complex involved in the docking of po lgi tra sport vesicles to sites of membrane remodeling during cellular processes s a a ization, migration, and division $[39,40]$. The exocyst complex is composed of 3, Sec5, vec6, Sec8, Sec10, Sec15, Exo70, and Exo84. Sec3 and Exo70 interact yith Rho small GTPases, which are key regulators of polarized cell growth in yeas ol Sec segarded as a spatial landmark for the development of polarity in budding st d in epithelial cells. This protein has been implicated in coordinating the membi geang of the remaining members of the exocyst complex that travel on secretory gran [43].

$\mathrm{Sec} 3$ is a docking protein of the exocyst cor that is re ared for secretion. The PHlike domain, which is formed by amino acids 1-1 the NH2-terminus, has the ability to bind PIP2, phospholipids, GTPases (Rho1/Cdc , , a he $\beta / \gamma$ subunit of G proteins [44]. Therefore, we also studied the Sec3 protein i rwa pithelial cells to evaluate whether Ezrin is associated with exocyst-related $p^{r}$ is. nofluorescence and immunoblotting assays showed that Sec3 was recrui a marily to the plasma membrane after NE stimulation, which is consisten with cal ation of Ezrin. This result is also consistent with those of another study [4 King reagent neomycin abrogated this effect. Furthermore, we used an Ezrin deletion $\left(\right.$ Ezrin $\left._{\triangle 1-333}\right)$ mutant in this study to confirm the roles of PIP2 in regulating the finct Ezrin. Immunoprecipitation experiments revealed that Ezrin and Sec3 com ${ }^{1}$ ad to form complexes; however, these complexes could not be detected in cells transfe d wir he Ezrin ${ }_{\triangle 1-333}$ mutant that lacks the PIP2 binding domain, even when exogenous $P$ added. These results demonstrate that Ezrin/Sec3 formed a complex and fy dogether, contributing to MUC5AC secretion. These proteins did not interact dir ly sered as a linker and was required for complex formation and for

In sy ary, resented evidence that the docking protein Sec3 is targeted to plasma $m e$ nane nains enriched in Ezrin after NE stimulation and that PIP2 is required for Se ding Ezrin. These events were followed by a higher level of MUC5AC secretion inton rnatant than occurs in normal static cells, indicating the involvement of these

prow in the mucus secretion process. The specific functions and co-localization of

F an and Sec3 provide new potential therapeutic targets for the intervention of mucus ayp secretion in airway diseases.



\section{Acknowledgments}

This study was supported by the National Natural Science Foundation of China (Grant No. 81100003, No. 81370111, and No. 31171346); the New Teachers' Fund for Doctor Station of the Ministry of Education, China (Grant No. 20115503120006); the Chongqing Nature Science Foundation (Grant No. cstc2011jjA10046); and Chongqing Medical Research Projects (Grant No. 20142029). 


\begin{tabular}{|c|c|c|}
\hline \multirow{2}{*}{$\begin{array}{c}\text { Cellular Physiology } \\
\text { and Biochemistry }\end{array}$} & \multirow{2}{*}{\multicolumn{2}{|c|}{ 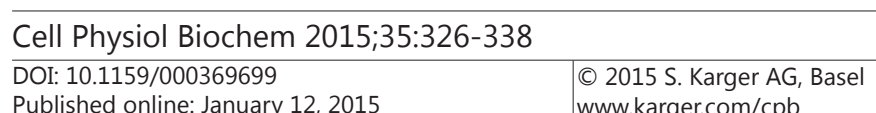 }} \\
\hline & & $\begin{array}{l}\text { O } 20115 \mathrm{~S} \text {. Karger AG, Basel } \\
\text { www.kargercom/cpb }\end{array}$ \\
\hline
\end{tabular}

\section{References}

1 Jackson AD: Airway goblet cell mucus secretion. Trends Pharmacol Sci 2001; 22: 39-45.

2 Williams OW, Sharafkhaneh A, Kim V, Dickey BF, Evans CM: Airway mucus: From production to secretion. Am J Respir Cell Mol Biol 2006;34:527-536.

3 Simpson JL, McDonald VM, Baines KJ, Oreo KM, Wang F, Hansbro PM, Gibson PG: Influence of Age, Past Smoking, and Disease Severity on TLR2, Neutrophilic Inflammation, and MMP-9 Levels in COPD. Mediators Inflamm 2013;2013:462934.

4 Tetley TD: Inflammatory cells and chronic obstructive pulmonary disease. Curr Drug Targets Inflamm Allergy 2005;4:607-618.

5 Gibson PG: Inflammatory phenotypes in adult asthma: clinical applications. Clin Respir J

6 Rogem DF, Barnes PJ: Treatment of airway mucus hypemecretion. Ann Med 2006;38:116-12

7 Rogers DF: The role of airway secretions in COPD: pathophysiology epidemiology an therapeutic options. COPD 2005;2:341-353. therapeutic options. COPD 2005;2:341-353.
Rogers DF: Physiology of airway mucus secretion and pathophysiology of hyperse trom
2007;52:1134-1146; discussion 1146-1149.

9 Stapleton G, Malliri A, Ozanne BW: Downregulated AP-1 activity is ascociated wh ibition of ProteinKinase-C-dependent CD44 and ezrin localisation and upregulatior cells. J Cell Sci 2002;115:2713-2724.

10 Barreiro 0, Yanez-Mo M, Serrador JM, Montoya MC, Vicente-Manzana H, Sanchez-Madrid F: Dynamic interaction of VCAM-1 and ICAM-1 wit sin and ezrin in a novel endothelial docking structure for adherent leukocyte ell Biol 2002; .1233-1245.

11 Zhang X, Orlando K, He B, Xi F, Zhang J, Zajac A, Guo W: rane association and functional regulation of Sec3 by phospholipids and Cdc42. J Cell Biol 2008;1

12 Barbers RG, Papanikolaou IC, Koss MN, Patel A, Kat: ra E nas M, Chan K, Azen CG, Sharma OP: Near fatal asthma: clinical and airway biopsy chara_tio Med 2012;2012:829608. 13 Kim S, Nadel JA: Role of neutrophils in mu ny secretion in COPD and implications for therapy. Treat
Respir Med 2004;3:147-159.

14 Kohri K, Ueki IF, Nadel JA: Neutr ast es mucin production by ligand-dependent epidermal growth factor receptor activatio $\quad$ Thysiol Lung Cell Mol Physiol 2002;283:L531-L540.

15 Roy C, Martin M, Mangeat P: A dual in ent the amino-terminal domain of ezrin in F- and G-actin binding. J Biol Chem 1997 20088-20095.

16 Osawa H, Smith CA, Ra Congk I P, Rutka JT: The role of the membrane cytoskeleton cross-linker ezrin in medulloblastoma cells $\quad$ col 2009;11:381-393.

17 Barret C, Roy C rrrer r, Mangeat P, Niggli V: Mutagenesis of the phosphatidylinositol 4,5-bisphosp inding site in the $\mathrm{NH}(2)$-terminal domain of ezrin correlates with its altered cellular rout r. ell Biol 2000;151:1067-1080.

18 Li Q 7 XD, Ko VP, Perelman JM: Nicotine suppresses inflammatory factors in HBE16 airway

"Is after exposure to cigarette smoke extract and lipopolysaccharide. Transl Res 2010;156:326-

19 Wuytack F, Raeymaekers L, De Smedt H, Casteels R: Polyamines and neomycin inhibit the pur. plasma-membrane Ca2+ pump by interacting with associated polyphosphoinositides. Biochem J 1. $89 ; 261: 1055-1058$.

40 oud B, Salminen A, Walworth NC, Novick PJ: A GTPbinding protein required for secretion rapidly associates with secretory vesicles and the plasma membrane in yeast. Cell 1989;53:753-768.

21 Kim D, Cavanaugh EJ, Simkin D: Inhibition of transient receptor potential A1 channel by phosphatidylinositol-4,5-bisphosphate. Am J Physiol Cell Physiol 2008;295:C92-C99.

22 Kim MH, Choi BH, Jung SR, Sernka TJ, Kim S, Kim KT, Hille B, Nguyen TD, Koh DS: Protease-activated receptor-2 increases exocytosis via multiple signal transduction pathways in pancreatic duct epithelial cells. J Biol Chem 2008;283:18711-18720.

23 Li M, Li Q, Yang G, Kolosov VP, Perelman JM, Zhou XD: Cold temperature induces_mucin_hypersecretion from normal human bronchial epithelial cells in vitro through a transient receptor potential melastatin 8 (TRPM8)-mediated mechanism. J Allergy Clin Immunol 2011;128:626-34.e1-5. 


\begin{tabular}{|c|c|c|}
\hline Cellular Physic & Cell Physiol Biochem 2015;35:326-338 & \\
\hline and Biochemistry & $\begin{array}{l}\text { DOI: 10.1159/000369699 } \\
\text { Published online: January 12, } 2015 \\
\end{array}$ & $\begin{array}{l}\text { O } 2015 \text { S. Karger AG, Basel } \\
\text { www.karger.com/cpb }\end{array}$ \\
\hline
\end{tabular}

24 Innes AL, Woodruff PG, Ferrando RE, Donnelly S, Dolganov GM, Lazarus SC, Fahy JV: Epithelial mucin stores are increased in the large airways of smokers with airflow obstruction. Chest 2006;130:1102-1108.

25 Li Q Zhou XD, Yu HM, Nie XH, Xu XY: Regulation of neutrophil elastase-induced MUC5AC expression by nuclear factor erythroid-2 related factor 2 in human airway epithelial cells. J Investig Med 2010;58:730736.

26 Groneberg DA, Eynott PR, Lim S, Oates T, Wu R, Carlstedt I, Roberts P, McCann B, Nicholson AG, Harrison BD, Chung KF: Expression of respiratory mucins in fatal status asthmaticus and mild asthma. Histopathology 2002;40:367-373.

27 Meyer ML, Potts-Kant EN, Ghio AJ, Fischer BM, Foster WM, Voynow JA: NAD(P)H quinone oxidoreduct 1 regulates neutrophil elastase-induced mucous cell metaplasia. Am J Physiol Lung Cell Mol Physiol 2012;303 L181-L188.

28 Marion S, Hoffmann E, Holzer D, Le Clainche C, Martin M, Sachse M, Ganeva I, Mangeat P, Grif Promotes Actin Assembly at the phagosome membrane and regulates phago-lysoson $f_{\mathrm{V}} \wedge$ ion. 2011;12:421-237.

29 Parisiadou L, Xie C, Cho HJ, Lin X, Gu XL, Long CX, Lobbestael E, Baekelandt V, Tayn $\mathrm{H}$ : Phosphorylation of ezrin/radixin/moesin proteins by LRRK2 promotes the $r$ ingemen. I actin cytoskeleton in neuronal morphogenesis. J Neurosci 2009;29:13971-13980.

30 Louvet-Vallée S: ERM proteins: from cellular architecture to cell sir ol Cell 2

31 Janke M, Herrig A, Austermann J, Gerke V, Steinem C, Janshoff A: Ac inu. in is activated by specific recognition of PIP2-functionalized lipid bilayers. Biochemist

32 Carvalho K, Khalifat N, Maniti O, Nicolas C, Arold S, Picart C, Ramos L: 4,5-bisphosphate-induced conformational change of and formatio ezrin oligomers. Biochemistry 2010;49:9318-9327.

33 Niggli V, Rossy J: Ezrin/radixin/moesin: versatile co cytoskeleton. Int J Biochem Cell Biol 2008;40:344-3

34 Maniti O, Khalifat N, Goggia K, Dalonneau F, G and ezrin to membranes containing phosp ady ositol $(4,5)$ bisphosphate: a comparative study of the affinity constants and conformational che 10 m Biophys Acta 2012;1818:2839-2849.

35 He H, Watanabe T, Zhan X, Huan uu. rami K, Takenawa T, Kumar CC, Simpson RJ, Maruta H: Role of Phosphatidylinositol 4,5 sphate in Ras/Rac-Induced Disruption of the Cortactin-Actomyosin II Complex and Malignant Trancform.

36 Zhu L, Crothers J Jr, Zhou re JG:A possible mechanism for ezrin to establish epithelial cell polarity. Am J Physiol Cell Physio 10;25 431-C443.

37 Zhou R, Zhu L, Kodani A, ao X, Forte JG: Phosphorylation of ezrin on threonine 567 produces a change in secre notypu and repolarizes the gastric parietal cell. J Cell Sci 2005;118:4381-4391.

38 Liu J, Guo W: ef mplex in exocytosis and cell migration. Protoplasma 2012;249:587-597.

39 Anderse $\mathrm{J}$, Yea in sec3-containing exocyst complex is required for desmosome assembly in mamr nepit cells. Mol Biol Cell 2010;21:152-164.

40 Z J X, . Zhang Y, Hsu SC, Zhou D, Guo W: Exo70 interacts with the Arp2/3 complex and regulates migra Nat Cell Biol 2006;8:1383-1388.

41 X ${ }_{j}$ E, Novick P, Du L, Kozminski KG, Lipschutz JH, Guo W: Cdc42 interacts with the exocyst and regu. polarized secretion. J Biol Chem 2001;276:46745-46750.

4. Morin A, Cordelières FP, Cherfils J, Olofsson B: RhoGDI3 and RhoG: Vesicular trafficking and interactions ith the Sec3 Exocyst subunit. Small GTPase 2010;1:142-156.

Boyd C, Hughes T, Pypaert M, Novick P: Vesicles carry most exocyst subunits to exocytic sites marked by the remaining two subunits, Sec3p and Exo70p. J Cell Biol 2004;167:889-901.

44 Lopez JP, Turner JR, Philipson LH: Glucose-induced ERM protein activation and translocation regulates insulin secretion. Am J Physiol Endocrinol Metab 2010;299:E772-E785.

45 Bendezú FO, Vincenzetti V, Martin SG: Fission yeast Sec3 and Exo70 are transported on actin cables and localize the exocyst complex to cell poles. PLoS One 2012;7:e40248. 